\title{
Neutrophil-derived APRIL concentrated in tumor lesions by proteoglycans correlates with human B-cell lymphoma aggressiveness
}

\author{
Juerg Schwaller, ${ }^{1}$ Pascal Schneider, ${ }^{2}$ Paulette Mhawech-Fauceglia, ${ }^{3}$ Thomas McKee, ${ }^{4}$ Samir Myit, ${ }^{4}$ \\ Thomas Matthes, ${ }^{5}$ Jurg Tschopp, ${ }^{1}$ Olivier Donze, ${ }^{6}$ Frederique-Anne Le Gal, ${ }^{7}$ and Bertrand Huard $8-10$ \\ ${ }^{1}$ Department of Research, University Hospital, Basel, Switzerland; '2Department of Biochemistry, University of Lausanne, Lausanne, Switzerland; ${ }^{3}$ Roswell Park \\ Cancer Institute, Buffalo, NY; ${ }^{4}$ Department of Pathology, University Medical Center, Geneva, Switzerland; ${ }^{5} \mathrm{Hematology}$ Unit, Department of Internal Medicine, \\ University Hospital, Geneva, Switzerland; ${ }^{6}$ Apotech Corp, Epalinges, Switzerland; ${ }^{7}$ Department of Dermatology, University Hospital, Geneva, Switzerland; \\ ${ }^{8}$ Louis Jeantet Laboratory, University Medical Center, Geneva, Switzerland; ${ }^{9}$ Department of Dermatology, University Hospital, Geneva, Switzerland; and \\ ${ }^{10}$ Department of Pathology-Immunology, University Hospital, Geneva, Switzerland
}

\begin{abstract}
A PRoliferation-Inducing TNF Ligand (APRIL) costimulates B-cell activation. When overexpressed in mice, APRIL induces B-cell neoplasia, reminiscent of human B-cell chronic lymphoid leukemia (B-CLL). We analyzed APRIL expression in situ in human non-Hodgkin lymphomas. APRIL up-regulation was only observed in high-grade B-cell lymphomas, diffuse large B-cell lymphoma (DLBCL), and Burkitt lymphoma (BL). Up-regulation was seen in $46 \%$ and $20 \%$ of DLBCL and
\end{abstract}

BL, respectively. In DLBCL, neutrophils, constitutively producing APRIL and infiltrating the tumor tissue, were the main cellular source of APRIL. Rare DLBCL cases showed a predominance of histiocytes or mesenchymal cells as APRIL source. APRIL secreted by neutrophils accumulated on tumor cells via proteoglycan binding. In addition to proteoglycans, DLBCL tumor cells expressed the APRIL signaling receptor, $\mathrm{TACl}$ and/or $\mathrm{BCMA}$, indicating that these tumor cells are fully equipped to respond to APRIL. A retrospective clinical analysis revealed a significant correlation between high expression of APRIL in tumor lesions and decreased overall patient survival rate. Hence, APRIL produced by inflammatory cells infiltrating lymphoma lesions may increase tumor aggressiveness and affect disease outcome. (Blood. 2007;109: 331-338)

(c) 2007 by The American Society of Hematology

\section{Introduction}

Non-Hodgkin B-cell lymphomas (NHLs) are divided into 3 prognostic groups designated low-, intermediate-, and highgrade B-cell lymphomas based on survival statistics, with high-grade B-cell lymphomas harboring the worse prognosis. High-grade lymphomas arising from mature B cells are constituted by Burkitt lymphoma (BL) and diffuse large B-cell lymphoma (DLBCL). The latter represents the most common form of adult NHL. DLBCL is widely accepted for its heterogeneity, best reflected by differences in clinical responses to chemotherapy. ${ }^{1}$ DLBCL heterogeneity was recently molecularly identified with gene-expression array studies. ${ }^{2}$ Despite the significant improvement achieved in the therapy of this disease with the emergence of B-cell-specific (anti-CD20) monoclonal antibody treatment, resistant DLBCL remain a significant clinical problem. Indeed, the recent report analyzing the effect of chemotherapy combined with anti-CD20 indicates that $40 \%$ of patients do not survive in a 5-year follow-up period. ${ }^{3}$ Future improvements to DLBCL therapy are therefore likely to result from the identification of key molecular targets in the treatmentresistant subtypes.

APRIL (also known as TALL-2) and BAFF (also known as BLys, TALL-1) are closely related ligands of the TNF superfamily. They share 2 receptors, BCMA and TACI, whereas BAFF binds to a third receptor BAFF-R (also known as BR3). ${ }^{4}$
BAFF-R, TACI, and BCMA are predominantly expressed on B cells, consistent with a role for these 2 TNF ligands in humoral immune responses. The BAFF/BAFF-R pathway is crucial for the maturation and maintenance of peripheral $B$ cells. ${ }^{5,6}$ BAFF participates also in humoral immune responses by providing B-cell costimulation and inducing Ig switch. ${ }^{7,8}$ APRIL has no obvious role in B-cell development ${ }^{9}$ but, like BAFF, is involved in humoral immune responses. ${ }^{7,8,10}$ Dysregulation of the BAFF/APRIL pathways has been associated with several autoimmune diseases. ${ }^{11}$

In addition to a role in autoimmune pathologies, APRIL and BAFF have been recently implicated in the development of tumors. ${ }^{12}$ In animal models, APRIL and BAFF overexpression induces development of B-cell neoplasia. ${ }^{13,14}$ The involvement of these 2 TNF ligands in B-cell tumors has been substantiated with in vitro studies on human cell lines. Exogenous APRIL confers a survival advantage to NHL, ${ }^{15-17}$ and multiple myeloma. ${ }^{18-20} \mathrm{Re}-$ cently, APRIL binding to the heparan sulfate side chains of proteoglycans (HSPG) was demonstrated. ${ }^{21,22}$ In contrast, BAFF does not bind HSPG, suggesting differences in APRIL and BAFF biology. Here, we analyzed in situ APRIL expression in human NHL. We observed that APRIL was only up-regulated in highgrade B-cell lymphomas and found that proteoglycans contribute to a tumor-promoting role of APRIL in these lymphomas.
Submitted February 3, 2006; accepted June 28, 2006. Prepublished online as Blood First Edition Paper, September 5, 2006; DOI 10.1182/blood2006-02-001800

The online version of this article contains a data supplement.
The publication costs of this article were defrayed in part by page charge payment. Therefore, and solely to indicate this fact, this article is hereby marked "advertisement" in accordance with 18 USC section 1734.

C) 2007 by The American Society of Hematology 


\section{Patients, materials, and methods}

\section{Cells and reagents}

All culture media were RPMI-1640 supplemented with 10\% FCS. The DLBCL lines SU-DHL-4 and -7 and OCI-Ly3, OCI-Ly7, and OCI-Ly10 were obtained from Dr A. Wiestner (NIH, Bethesda, MD). Dendritic cells were obtained from peripheral monocytes treated with GM-CSF/IL-4 as previously described. ${ }^{23}$ Granulocytes were obtained from bone marrow $\mathrm{CD}_{3} 4^{+}$cells as previously reported. ${ }^{24}$ Polymorphonuclear cells (PMNs) were isolated from healthy donors. Briefly, blood was collected on sodium citrate $(3.8 \%)$. Red blood cells were removed by sedimentation on PBS Dextran T500, and floating cells were further separated by centrifugation on a layer of Ficoll-Hypaque. Pelleted cells were PMNs, whereas layered cells were peripheral-blood mononuclear cells (PBMCs). To avoid PMN activation, all separation steps were performed at $4^{\circ} \mathrm{C}$ in the presence of $5 \mu \mathrm{g} / \mathrm{mL}$ Polymixin B. Heparin (Liquemin, $5000 \mathrm{IU} / \mathrm{mL}$ ) was from Roche Pharma (Basel, Switzerland). Anti-TACI (clone 1A1, rat IgG2a,), anti-BCMA (clone Vicky1, rat IgG2a), anti-APRIL against the extracellular portion (93-233) of APRIL (clones Aprily-1, -2, -4, -5, -6, -8, all mouse IgG1), and pAb ED (rabbit polyclonal against the aa 67-79 peptide GTGGPSQNGEGYP of APRIL), called Stalk-1 hereafter, were obtained from Alexis Biochemicals (Basel, Switzerland). An antiserum against the same extracellular portion (88-233) of APRIL was made in rabbit and used for detection in enzyme-linked immunoabsorbent assay (ELISA). MegaAPRIL consisting of extracellular APRIL (88-233) fused to ACRP30 (16-108), its control headless ACRP30, hereafter-called acrpAPRIL and acrpCTRL, respectively, were also from Alexis Biochemicals. Anti-CD15 (clone C3D-1, IgM), antielastase (clone NP57, IgG1), anti-CD20 (clone L26, IgG2a), anti-bcl-6 (clone PG-B6P, IgG1), anti-Ki-67 (clone MIB-1, IgG1), antivimentin (clone V9, IgG1), anti-CD68 (clones PGM-1 and KP1), and anti-L1 protein (clone Mac 387, IgG1) antibodies were from Dako (Glostrup, Denmark). Anti-CD10 (clone 56C6, IgG1) was from Novacastra (Newcastle upon Tyne, United Kingdom). Anti-IRF-4 (goat polyclonal) and anti-syndecan-4 (rabbit polyclonal) were from Santa Cruz Biotechnology (Santa Cruz, CA). The anti-CD10 used to stain bone marrow granulocytes in flow cytometry was from Pharmingen (San Diego, CA). Expression vectors for Flag ligands and receptors fused to the cartilage outer membrane protein (COMP) have been previously described..$^{25,26}$ An APRIL bearing the mutation $\mathrm{RR}_{103-104}$ to $\mathrm{AA}$ was created by polymerase chain reaction

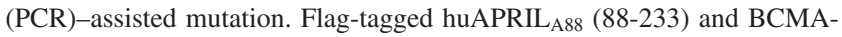
COMP were produced by transiently transfected $293 \mathrm{~T}$ cells in serum-free optimem (Gibco BRL, Rockville, MD) and purified by M2-Sepharose affinity chromatography (Sigma, St Louis, MO).

\section{Flow cytometry}

Cell staining was performed as previously described. ${ }^{23}$ Antibody staining was revealed with a secondary Alexa-488-conjugated goat anti-mouse or anti-rabbit serum (Molecular Probes, Leiden, The Netherlands). Ligand staining was revealed with an anti-Flag antibody (IgG1, Clone M2; Sigma) at $5 \mu \mathrm{g} / \mathrm{mL}$ and phycoerythrin-conjugated goat-anti-mouse IgG1 (Jackson Immunoresearch, Baltimore, PA). For total staining, cells were fixed/ permeabilized with PBS $1 \%$ formaldehyde, and staining was performed in the presence of $1 \%$ saponin.

\section{Detection of secreted APRIL}

For APRIL quantification in sera, BCMA-Ig $1 \mu \mathrm{g} / \mathrm{mL}$ (Alexis Biochemicals) was used as capture, and detection was performed with a homemade rabbit polyclonal anti-huAPRIL $(1 \mu \mathrm{g} / \mathrm{mL})$, followed by an HRPconjugated goat anti-rabbit IgG serum (Jackson Immunoresearch). Tetramethylbenzidine (Sigma) was used as substrate for HRP. Sensitivity of this ELISA was 1 to $3 \mathrm{ng} / \mathrm{mL}$.

For APRIL detection in cell supernatants, secreted APRIL was immunoprecipitated with BCMA-Ig and detected in Western blot with the Aprily-1 mAb as previously described. ${ }^{23}$ Supernatant $(5 \mathrm{~mL})$ was conditioned by $50 \times 10^{6}$ neutrophils pooled from 5 donors in the presence of $1000 \mathrm{IU} / \mathrm{mL}$
G-CSF (kind gift of V. Kindler, Geneva, Switzerland) for 48 hours. Control supernatant was conditioned by $50 \times 10^{6} \mathrm{PBMCs}$ from a single donor.

\section{Gene-expression analysis}

Total RNA (25 ng) prepared from cells using TRIzol Reagent (Gibco BRL) was reverse transcribed and amplified using a one-step reverse transcriptase (RT)-PCR kit (Qiagen, Chatsworth, CA). All the primers used in this study spanned intronic sequences on the genomic DNA. For hTACI 5'ctgggtacctgcatgtcctg- $3^{\prime}$ and $5^{\prime}$-agacttggccggacttgac- $3^{\prime}$, for hBCMA $5^{\prime}$ gggcagtgctcccaaaat- $3^{\prime}$ and $5^{\prime}$-tcgttttcgtggtgacaaga- $3^{\prime}$, and for hAPRIL $5^{\prime}$-atgccagcctcatctccttt- $3^{\prime}$ and $5^{\prime}$-tcctggattcggacaccata- $3^{\prime}$ were used as forward and reverse primers, respectively. The control actin primers were forward $5^{\prime}$-ttaacgagaagctgtgctacgtc- $3^{\prime}$ and reverse $5^{\prime}$-atagtcetgcttgcttgctgatccac- $3^{\prime}$. Amplicon specificity was ascertained by restriction analysis with 3 selected enzymes for each primer pair.

\section{Immunohistochemistry}

For immunocellularchemistry (ICC), cells were injected into murine intestine. The injected intestines were fixed and embedded into paraffin. The resulting blocks were then treated as a standard tissue for immunostaining.

Patient's diagnosis was made according to the World Health Organization classification. Tissue microarrays were constructed as previously described..$^{27}$ For immunoperoxidase staining, secondary reagents were goat Ig anti-rabbit or anti-mouse IgG conjugated to HRP (Jackson Immunoresearch), followed by streptABComplex/HRP (Dako) and 3-amino-9ethylcarbazole substrate (Sigma). For 2-color immunofluorescence stainings, isotype-specific goat antimouse sera conjugated to biotin or phycoerythrin (Jackson Immunoresearch) and goat Ig anti-rabbit IgG conjugated to Alexa-488 (Molecular Probes) were used. For light and fluorescence microscopy, mounting media were aquatex (Merck, GlattbruggOpfikon, Switzerland) and 12\% Molwiol 4-88, 30\% glycerol in $0.2 \mathrm{M}$ Tris, $\mathrm{pH}$ 8.5. Light and fluorescence microscopy images were visualized with an Axiophot I microscope (Carl Zeiss, Jena, Germany) equipped with one of the following: a Plan Neofluar $63 \times / 1.25$ oil-immersion objective lens (Figures 2 and 3I), a Plan Neofluar $100 \times / 1.30$ oil-immersion objective lens (Figure 3E-H), or a Plan Neofluar $40 \times / 0.75$ air objective (Figures 3A-D and 5A-C). Images were captured with an Axiocam color CCD camera and were treated on a Pentium III computer with Axiovision software (Carl Zeiss). Confocal analysis was performed on an LSM 510 microscope (Carl Zeiss) equipped with a Plan Neofluar $40 \times / 1.30$ oil-immersion objective lens (Figure 3J).

\section{Patient follow-up and statistical analysis}

All patients with a diagnosed DLBCL were seen, and their specimen was obtained between 1995 and 2003 at the Geneva University Hospital. Retrospective study of the clinical data from these patients was performed. Patients provided written informed consent in accordance with the Declaration of Helsinki. The project was reviewed and approved by the Geneva University Hospital Ethics Committee. Survival was measured from the date of biopsy until the date of death from lymphoma, excluding comorbidity. Patients alive at the last follow-up evaluation were censored (tickles on the curves). Kaplan-Meier overall survival curve was performed with Prism software (GraphPad Software, San Diego, CA). Comparison between groups was based on a log-rank statistical test.

\section{Results}

Characterization of antibodies selectively recognizing secreted APRIL and APRIL-producing cells

To study in situ APRIL expression, APRIL-specific antibodies with different specificities were selected. The mAb Aprily-2 was raised against the c-terminal TNF homology domain of APRIL, secreted on furin cleavage. The polyclonal rabbit antiserum Stalk-1 was raised against a peptide in the membrane-proximal part of APRIL 


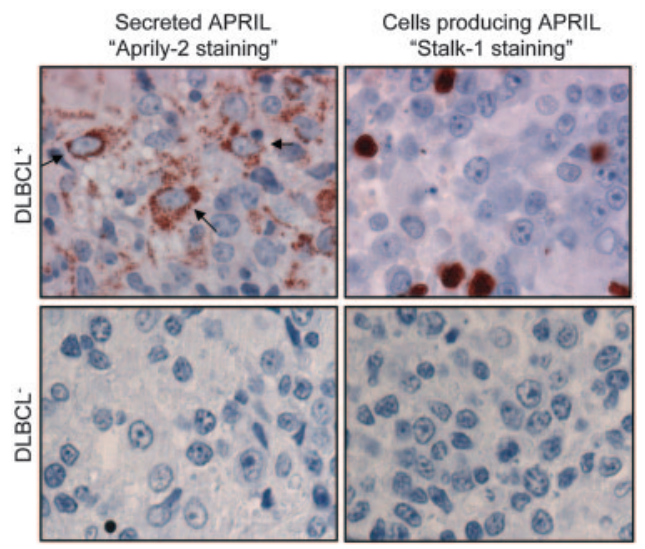

Figure 1. APRIL is strongly expressed in DLBCL lesions. DLBCL tumor lesions were stained with Aprily-2 (left) and Stalk-1 (right). Representative tumor lesions with high (top) and low (bottom) expression of APRIL are shown. Original magnification was $\times 63$. Concentration of the Aprily- 2 staining on cells with a morphology reminiscent of DLBCL was marked with an arrow.

extracellular domain remaining associated to the cell membrane after furin cleavage. This domain is called the APRIL Stalk hereafter. Selective stainings on transfected cells overexpressing APRIL were obtained with these antibodies (Figure S1A, available on the Blood website; see the Supplemental Figures link at the top of the online article). As described below, Stalk-1 and Aprily-2 generated positive staining by immunohistochemistry (IHC) on DLBCL lesions (Figure 1). The protein products detected by Stalk-1 and Aprily-2 were biochemically characterized from lysates of these tumor lesions. Western blot analysis showed that Aprily-2 identified a predominant $18-\mathrm{kDa}$ band in IHC-positive lesions, whereas no such band was observed in IHC-negative lesions (Figure 2A, left). The $18-\mathrm{kDa}$ band recognized by Aprily-2 is compatible with the size of secreted APRIL that we previously described in the supernatant of dendritic cells. ${ }^{23}$ Notably, the full-length molecule $(30 \mathrm{kDa})$ was not detected in lesions. This absence of detection was not due to a failure of Aprily-2 to recognize full-length APRIL, because Aprily-2 stained 293T transfected cells expressing an uncleaved mutant of APRIL in ICC (Figure S1B). In contrast, Stalk-1 recognizes a 14-kDa band selectively in IHC-positive lesions (Figure 2A, middle), compatible with the predicted size of the APRIL stalk domain after cleavage. This cleaved product is also observed as a predominant band in dendritic-cell (DC) lysates (Figure 2A, right). Specificity of the Abs was further assessed in competition experiments. Addition of soluble APRIL or stalk peptide to the respective antibodies prior to Western blot analysis abolished the recognition of the $18-\mathrm{kDa}$ and $14-\mathrm{kDa}$ bands, respectively (Figure 2B), demonstrating that the molecular species recognized in Western blot expressed the relevant epitopes. The combined absence of detection of the full-length product by these 2 antibodies shows that most of APRIL is rapidly cleaved and secreted after synthesis in APRIL-producing cells. This observation is consistent with the early APRIL processing in the secretory pathway described previously. ${ }^{28}$ It also indicates that the stalk of cleaved APRIL remains stable in APRIL-producing cells, making Stalk-1 a valuable tool to detect APRIL-producing cells, whereas Aprily-2 detects secreted APRIL.

\section{Strong up-regulation of APRIL in DLBCL lesions}

The Aprily-2 mAb was used to stain multitumor arrays prepared from NHL lesions and control healthy tissues. Among the DLBCLs analyzed $(\mathrm{n}=56), 46 \%$ were strongly positive for secreted APRIL (Aprily-2 staining) (Figure 1, top). The remaining DLBCL cases showed only a focal staining pattern. The APRIL up-regulation was also seen in BL lesions (2 of 10). In contrast, all the lesions from low to intermediate NHL revealed only focally distributed stained cells with large areas of unstained cells as shown in Figure 1 (bottom). This was the case with mucosa-associated lymphoid tissue $(n=18)$, follicular-cell $(n=35)$, mantle-cell $(n=5)$, and marginal-zone $(n=6)$ lymphomas, as well as nodular chronic lymphocytic leukemia $(n=14)$. In a substantial proportion of these lesions, Aprily-2 staining was even undetectable. In DLBCL, lesions harboring a strong staining for secreted APRIL also contained abundant cells producing APRIL (Stalk-1 staining) (Figure 1, top). Hence, detection of secreted APRIL correlated with the presence of APRIL-producing cells in the lesions. Detailed analysis of the staining patterns revealed dissimilarity between Aprily-2 and Stalk-1. Although some Aprily-2 staining appears not to be associated to any cell, a majority of the staining was concentrated on cells with a morphology reminiscent of DLBCL. The punctuated Aprily-2 staining was also observed with 2 other mAbs raised against secreted APRIL (Aprily-6 and Aprily-8) (data not shown). In contrast, Stalk-1 produced a staining associated to cells with an apparent different morphology. In this experiment, Aprily-2 and Stalk-1 tissue staining was competed with soluble APRIL and stalk peptide, respectively, further demonstrating specificity (data not shown). Taken together, this IHC analysis indicates that APRIL expression is markedly up-regulated in some high-grade NHL lymphomas, such as DLBCL and BL, but not in lower grade NHLs. Once secreted, cleaved APRIL dissociates from APRIL-producing cells.

\section{Tumor-infiltrating neutrophils produce APRIL}

We next identified APRIL-producing cells in situ with 2 color immunofluorescence stainings on whole tumor sections. In 19 of 25
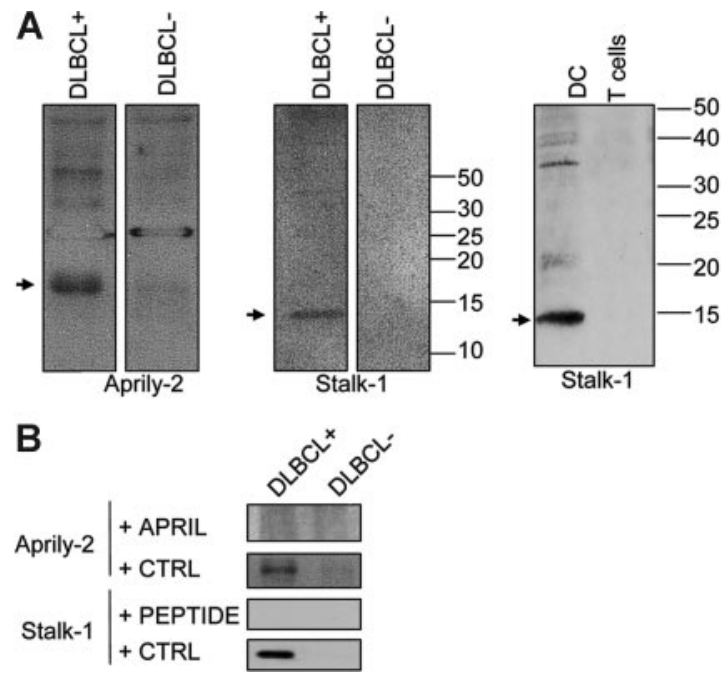

Figure 2. Characterization of anti-APRIL antibodies identifying secreted APRIL and cells producing APRIL. (A) Extracts $(20 \mu \mathrm{g})$ from IHC-positive and -negative DLBCL tissue samples were analyzed by Western blot with Aprily-2 $(2 \mu \mathrm{g} / \mathrm{mL})$ and Stalk-1 (5 $\mu \mathrm{g} / \mathrm{mL})$. An 18-kDa band corresponding to secreted APRIL was revealed with Aprily-2 (left), whereas Stalk-1 identified a 14-kDa band (middle). Similar $14-\mathrm{kDa}$ reactivity was observed with Stalk-1 on DC lysates $(20 \mu \mathrm{g})$ (right). The reactivity is representative of $3 \mathrm{IHC}$-positive and $2 \mathrm{IHC}$-negative tumor lysates. (B) Western blot specificity was ascertained in blocking experiments. Aprily-2 was preincubated with $10 \mu \mathrm{g} / \mathrm{mL}$ acrpAPRIL or acrpCTRL. Stalk-1 was preincubated with $10 \mu \mathrm{g} / \mathrm{mL}$ stalk peptide or an irrelevant peptide prior to Western blot analysis. 


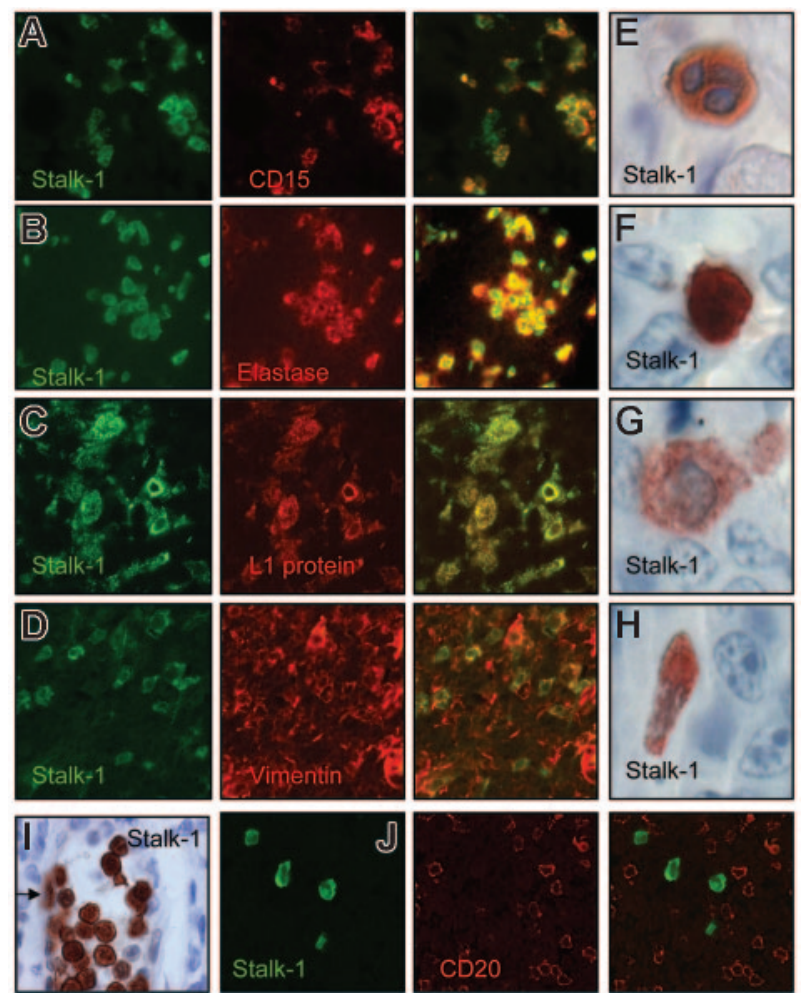

Figure 3. APRIL is produced mainly by neutrophils infiltrating DLBCL lesions. Stalk-1 staining (green) was combined to (A) anti-CD15 (1 of 50), (B) antielastase $(1 \mu \mathrm{g} / \mathrm{mL}),(C)$ anti-L1 protein $(10 \mu \mathrm{g} / \mathrm{mL})$, and (D) antivimentin $(5 \mu \mathrm{g} / \mathrm{mL})$ stainings (all in red). Single stainings and corresponding merge pictures shown are representative of 2 or more patients with DLBCL. (E) Morphology of Stalk-1-stained cells. (F-H) Intensity of Stalk-1 staining is shown for a cell with a morphology of $(F)$ a neutrophil, (G) a histiocyte, and $(\mathrm{H})$ a mesenchymal cell from the same lesion. The pictures were taken with the same exposure time. (I) The picture shows endothelial cells lining tumor-draining vessels (arrow) and blood neutrophils stained with Stalk-1.(J) Stalk-1 staining (green) was combined to anti-CD20 staining (red, $5 \mu \mathrm{g} / \mathrm{mL}$ ) and analyzed by confocal microscopy. Single stainings and corresponding merge picture are representative of 6 patients with DLBCL.

of the patients with DLBCL analyzed, greater than $60 \%$ of Stalk-1-positive cells expressed CD15, identifying them as granulocytes (Figure 3A). Their precise neutrophil identity was obtained by finding that they expressed elastase, a neutrophil-specific protease (Figure 3B) and by their characteristic nuclear morphology (Figure 3E). The neutrophil identity was confirmed because no eosinophil (eosin staining) or basophil (IgE staining) was detected in these lesions (data not shown). In the other patients, the Stalk-1-positive cells were histiocytes expressing the L1 protein (2 of 25; Figure 3C) or mesenchymal cells expressing vimentin (4 of 25; Figure 3D). L1-protein or vimentin-expressing cells observed here did not express CD68, excluding them from bona fide tumor-infiltrating macrophages (data not shown). In this experiment, we noticed that the intensity of the Stalk-1 staining in IHC was higher in neutrophils than in histiocytes and mesenchymal cells present in the same section (Figure 3F-H). Finally, we also observed few endothelial cells, lining vessels draining the tumor, also stained with Stalk-1 (Figure 3I). All CD15 ${ }^{+}$(or elastase $^{+}$) and L1 protein $^{+}$cells were stained with Stalk-1 (Figure 3A-C), indicative of a constitutive APRIL expression in neutrophils and histiocytes from DLBCL lesions. This was not the case for mesenchymal cells, because vimentin ${ }^{+}$Stalk-1 ${ }^{-}$cells were observed (Figure 3D). Taken together, this indicates that the cellular source of APRIL in DLBCL lesions is disparate, but neutrophils were the main source of APRIL. This was due to their abundant presence and their high level of APRIL production. As a consequence, DLBCL cases with APRIL up-regulation (Stalk-1 and Aprily-2 staining) correlated with high numbers of neutrophils. There was only one exception, that of a case containing histiocytes and harboring APRIL up-regulation. The other "histiocytic" case and the "mesenchymal" cases were low in APRIL expression. CD20 staining, used here as a DLBCL marker, was combined to Stalk-1 staining to localize APRIL-producing neutrophils in the lesions. Figure 3J shows that APRIL-producing cells were located close to tumor cells. APRIL-producing neutrophils were also observed inside (Figure 3I) or close to vessels (data not shown), indicating an extra vasation from blood vessels draining the tumor.

To further characterize APRIL expression, neutrophils from healthy volunteers were studied. In this experiment, availability of fresh cells allowed us to confirm APRIL mRNA expression by circulating neutrophils (Figure 4A). APRIL protein was detected in isolated peripheral-blood neutrophils by ICC and in bone marrow resident cells with a morphology consistent with granulocytes by IHC (Figure S2). The reactivity was similar to in situ IHC with an absence of reactivity for Aprily- 2 on Stalk- $1^{+}$cells. APRIL protein expression in neutrophils was further studied by flow cytometry. Stalk-1 stained peripheral-blood and bone marrow CD10 ${ }^{+}$granulocytes after cell permeabilization (Figure 4B-C). No staining was observed with Aprily-2 in this assay. However, 3 independent mAbs (Aprily-4, -5, -8), also directed against the secreted domain, gave a staining (Figure 4B). The staining was weak, explaining the absence of detection in APRIL-producing cells by IHC or ICC. In contrast, there was no detectable staining at the cell surface with Stalk-1 or Aprily-4, -5 , or -8 by flow cytometry. Interestingly, we observed that APRIL expression was induced early in the differentiation of granulocytes, because the major fraction of high forward scatter $\mathrm{CD}^{-} 0^{-}$cells, representing immature cells, ${ }^{29}$ was also stained with Stalk-1 (Figure 4C, bottom). There was no reactivity
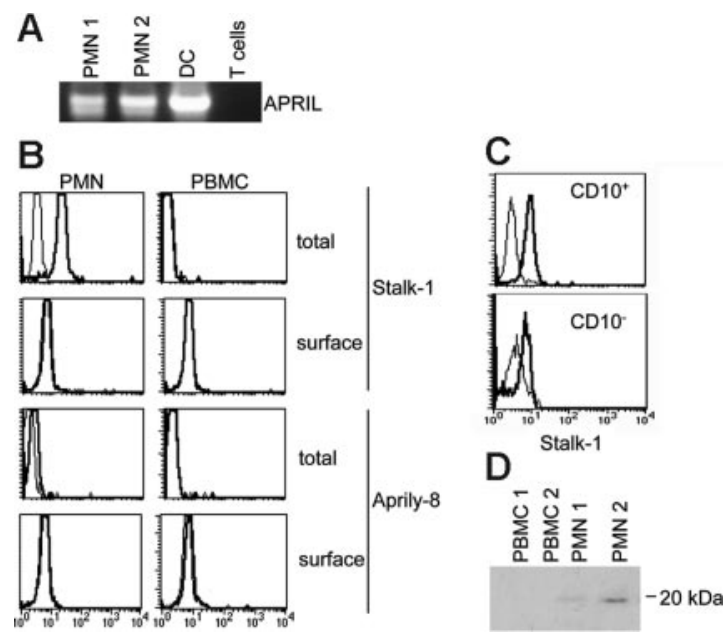

Figure 4. APRIL production by neutrophils is constitutive. (A) APRIL expression in peripheral neutrophils was observed at the gene-expression level by RT-PCR analysis. DC and T cells were used as positive and negative controls, respectively. (B) Total staining after cell permeabilization or surface staining was performed with Stalk-1 $(2 \mu \mathrm{g} / \mathrm{mL})$ and Aprily-8 $(1 \mu \mathrm{g} / \mathrm{mL})$ Abs. Histogram plots after flow cytometry analysis are shown. Thick and thin lines correspond to indicated Abs and isotypematched control Abs, respectively. Stainings are representative of 4 donors. (C) Bone marrow cellular suspensions were stained with Stalk-1 and anti-CD10 Abs. Histogram plots after flow cytometry analysis are shown on $\mathrm{CD}_{10}{ }^{+}$and $\mathrm{CD} 10^{-}$size-gated granulocytes (right). Thick and thin lines correspond to Stalk-1 and isotype-matched control Abs, respectively. Stainings are representative of 3 donors. (D) Supernatants conditioned by PMNs and PBMCs were immunoprecipitated with BCMA-Ig and analyzed by Western blot with the Aprily-1 mAb. 
on peripheral blood and bone marrow mononuclear cells, indicating that neutrophils were also the main source of APRIL in healthy tissues. Taken together, these analyses confirm the rapid APRIL processing, rendering the secreted domain of APRIL barely detectable in producing cells. In fact, the secreted domain was only detected in producing cells when APRIL was overexpressed (see Figure S1). They further indicate that the Stalk domain locates in the cytoplasm after APRIL processing. The precise cellular localization of the Stalk domain was analyzed by confocal microscopy. Costaining in granulocytes with Stalk-1 and CD15, a marker expressed at the cell membrane and into cytoplasmic granules, ${ }^{30}$ revealed that only a minor fraction of the Stalk domain was expressed at the cell surface. Most of it was observed diffusely in the cytoplasm (Figure S3, top). This distribution was confirmed by colocalization of Stalk-1 and the L1 protein, a marker expressed in the cytoplasm of histiocytes ${ }^{31}$ (Figure S3, bottom). We next tried to detect secreted APRIL. APRIL secretion was only detected in the supernatant of G-CSF-stimulated cells by immunoprecipitation followed by Western blot revelation (Figure 4D). The faint band observed gave an in vitro secretion estimate of less than 100 $\mathrm{pg} / \mathrm{mL} / 10 \times 10 \mathrm{e}^{6}$ cells $/ 48$ hours in this experiment. This weak secretion level explained our failure to detect secreted APRIL by sandwich ELISA (sensitivity ranging between 1 and $3 \mathrm{ng} / \mathrm{mL}$; data not shown). In conclusion, APRIL expression is induced early during neutrophil differentiation and sustained in mature neutrophils present in the bone marrow and circulating in peripheral blood. The level of expression between these latter cells and tumor-infiltrating neutrophils appears similar by IHC, indicating that APRIL expression by neutrophils is mainly constitutive.

\section{Proteoglycans ensure APRIL retention in lesions and binding onto tumor cells}

APRIL was recently shown to interact with cell-surface HSPG. ${ }^{21,22}$ We therefore assessed HSPG expression in DLBCL lesions. We observed expression of syndecan- 4 in some of the DLBCL cases analyzed, consistent with the fraction of DLBCLs expressing syndecan-4 mRNA. ${ }^{32}$ Remarkably, secreted APRIL is strictly located at syndecan-4 expression sites in the tumor lesions expressing this HSPG (Figure 5A). Such an overlap is strongly

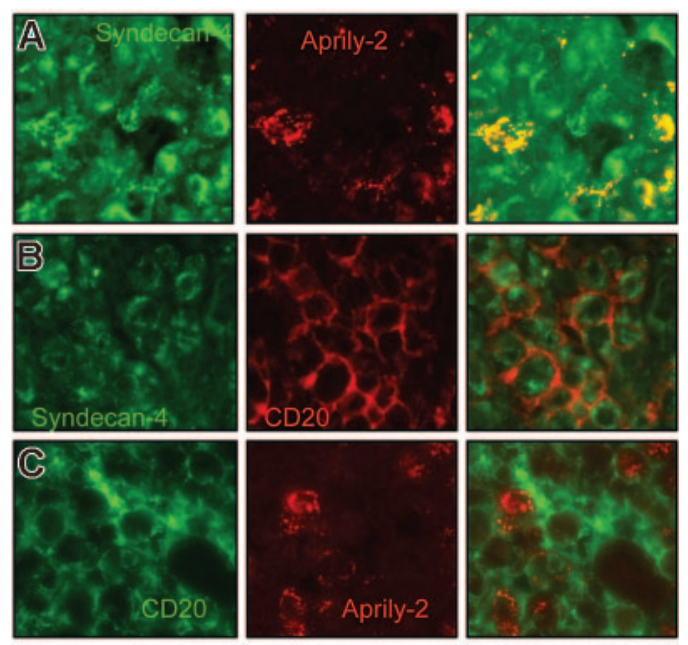

Figure 5. APRIL is concentrated on tumor cells by proteoglycans. (A) DLBCL lesions were stained with anti-syndecan-4 (green, $10 \mu \mathrm{g} / \mathrm{mL}$ ) and Aprily-2 (red), (B) with anti-syndecan-4 (green) and anti-CD20 (red), and (C) with anti-CD20 (green) and Aprily-2 (red). Single stainings and the corresponding merge picture are representative of 4 patients. suggestive of a tight interaction of APRIL with syndecan-4 in situ. In the other DLBCL cases, syndecan-4 was not expressed, but the Aprily-2 staining observed was identical, with characteristic punctuation, indicative of an association with HSPG from other(s) proteoglycan(s). Combination of CD20 and syndecan-4 staining showed that tumor cells homogeneously expressed syndecan-4 in this lesion, with a major pool expressed intracellularly compared with the CD20 membrane staining (Figure 5B). Finally, combination of CD20 and Aprily-2 showed that some of the tumor cells bound secreted APRIL, with also a major intracellular pool (Figure 5C). These analyses show that APRIL, once secreted by neutrophils, binds to the tumor cell via an interaction with HSPG.

We next tested APRIL up-regulation in the circulation. Sera from patients with "high APRIL expressor" DLBCL $(n=5)$ were tested for the presence of APRIL by ELISA. Circulating APRIL was not detected in these patients, indicating a concentration below $1 \mathrm{ng} / \mathrm{mL}$, whereas it was detected in sera from autoimmune patients with lupus erythrematosus ( $30 \mathrm{ng} / \mathrm{mL}$; data not shown), as previously reported. ${ }^{11}$ The up-regulation of APRIL is therefore local in DLBCL and does not reach the circulation, likely because of retention by proteoglycans in the tumor lesion.

\section{DLBCL tumor cells express BCMA and TACI in addition to proteoglycan}

We next assessed the expression of BCMA and TACI in DLBCL. Despite tests with several reactive antibodies, endogenous BCMA or TACI was not detected in situ in DLBCL tumor lesions, probably reflecting a low level of endogenous expression (data not shown). We therefore used DLBCL cell lines to assess APRIL receptor expression in tumor cells. RT-PCR analysis showed TACI and BCMA mRNA expression in 2 of 5 and all DLBCL cell lines, respectively (Figure 6A). In this experiment, we confirmed that DLBCLs do not express APRIL at the mRNA level compared with myeloid cells such as DCs, because an APRIL band was observed in DCs but not in DLBCLs even after 35 RT-PCR cycles. Anti-TACI and BCMA were next used. The specificity of these mAbs was first validated on transfected cells (Figure 6B). TACI protein expression at the cell surface was confirmed in the 2 DLBCL cell lines positive for TACI mRNA but not in the other lines. In contrast, expression of surface BCMA was barely detectable, but significant staining was observed after cell permeabilization in 3 of 5 DLBCL cell lines. The level of intracellular BCMA for the 2 other cell lines was weaker. Binding of soluble APRIL was next tested. Heparin and soluble BCMA were used to compete APRIL/HSPG and APRIL/TACI BCMA interactions as previously described. ${ }^{21}$ Soluble APRIL bound homogeneously to all DLBCL cell lines. However, the level of inhibition with heparin and soluble BCMA varied. Complete and absence of inhibition was observed on cell lines negative for TACI with heparin and BCMA, respectively, indicating that HSPG was the unique APRIL-R on the surface of these cells. In contrast, partial inhibition was seen with heparin and BCMA on the TACI-expressing cell lines, indicating that both TACI and HSPG were expressed as APRIL-R on the surface on these cells. Figure $6 \mathrm{C}$ shows APRIL receptor expression for the 2 representative cell lines OCI-Ly3 and OCI-Ly7. Two phenotypes for DLBCLs therefore exist regarding APRIL receptor expression, TACIsurf ${ }^{+}$BCMAint $^{\text {low }}$ HSPGsurf $^{+}$and TACIsurf $^{-}$BCMAint ${ }^{+}$HSPGsurf ${ }^{+}$. HSPG expression in association with TACI and/or BCMA expression was also observed in the BL cell lines Namalwa, Ramos and BL41 (data not shown). As previously reported by $\mathrm{He}$ et al, ${ }^{16}$ we also observed a survival signal in vitro 


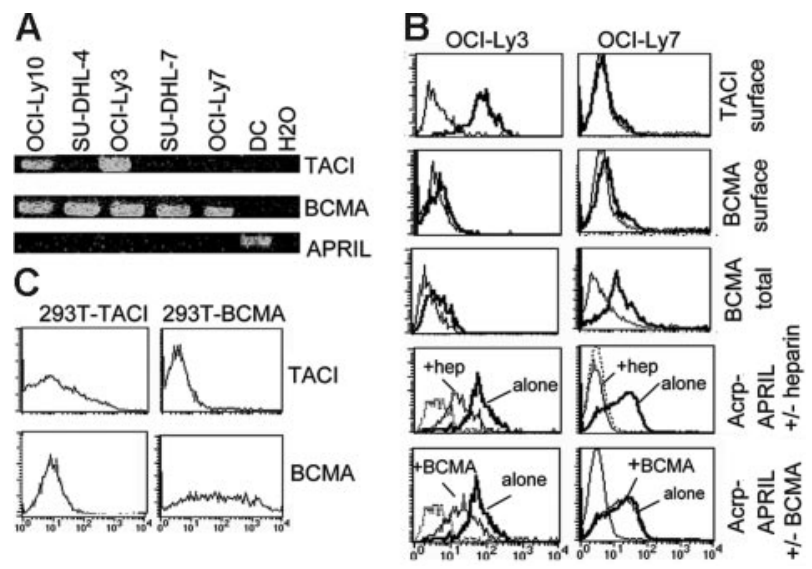

Figure 6. DLBCL tumor cells express $\mathrm{BCMA} / \mathrm{TACl}$ signaling receptor and proteoglycan coreceptor. (A) RT-PCR analysis of TACI, BCMA, and APRIL mRNA expression in DLBCL cell lines. (B) Specificity of the anti-TACI and BCMA mAbs is shown on transfected 293T cells. (C) APRIL-R expression analysis by flow cytometry. Control stainings (isotype control and acrpCTRL) are shown as dotted lines. Heparin and BCMA-COMP were used at $1 / 100$ and $50 \mu \mathrm{g} / \mathrm{mL}$, respectively. SUDHL-4 and -7 harbored identical staining to OCl-Ly7. OCl-Ly10 harbored an identical staining to OCl-Ly3.

for DLBCL with exogenous ACRP-APRIL (data not shown). Hence, DLBCLs are fully equipped to respond to APRIL signaling.

\section{High APRIL expression in tumor lesions of patients with DLBCL is associated with a reduced survival rate}

The role of APRIL in DLBCL was assessed by a retrospective clinical analysis of the patients analyzed here for APRIL expression in biopsy samples. Data on stage and LDH levels were available for a limited number of patients. High and low levels of APRIL could be observed for different stages and LDH expression (Table 1). There were also no significant differences between high and low APRIL expressors in terms of tumor proliferation index and age. In addition, low and high levels of APRIL expression were observed in the 2 DLBCL subtypes, germinal center and activated, indicating no obvious correlation between levels of APRIL expression and standard clinical parameters at the time of diagnosis. We next analyzed the survival rate. Of the 39 patients analyzed for APRIL expression, 34 had trackable survival data. Interestingly, a significant decrease $(P=.02)$ in survival was observed in the high

Table 1. Clinical data from patients with DLBCL

\begin{tabular}{|c|c|c|}
\hline & APRIL high & APRIL low \\
\hline \multicolumn{3}{|l|}{ Disease stage $^{\star}$} \\
\hline $\mathrm{I}, \mathrm{n}$ & 1 & 2 \\
\hline II, n & 1 & 2 \\
\hline III/IV, n & 4 & 4 \\
\hline \multicolumn{3}{|l|}{ Serum LDH level† } \\
\hline High, $n$ & 5 & 6 \\
\hline Low, $\mathrm{n}$ & 3 & 2 \\
\hline Proliferation index $\neq$, mean $\pm S D$ & $75 \pm 8(n=22)$ & $68 \pm 19(n=25)$ \\
\hline \multicolumn{3}{|l|}{ DLBCL type§ } \\
\hline $\mathrm{GC}, \mathrm{n}$ & 19 & 17 \\
\hline Activated, $\mathrm{n}$ & 17 & 10 \\
\hline Age, y, mean $\pm S D$ & $61 \pm 19(n=26)$ & $57 \pm 8(n=30)$ \\
\hline
\end{tabular}

LDH indicates lactate dehydrogenase; GC, germinal center.

*Disease stage was defined according to the Ann Arbor staging system.

†Serum LDH level was determined by standard techniques.

¥The proliferation index was determined by $\mathrm{Ki}-67$ in situ staining

$\S$ The DLBCL type was defined in IHC with the combination of CD10, bcl-6, and IRF-4 markers as reported recently by Hans et al. 46

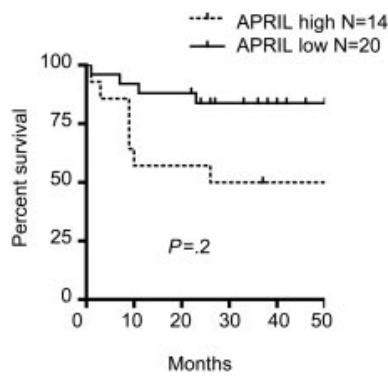

Figure 7. High APRIL expression in patients with DLBCL is associated with decreased survival. Survival was retrospectively studied in patients stratified according to APRIL expression.

APRIL expressor group (Figure 7). The survival rate at 5 years was $50 \%$ and $80 \%$ for the patients with high and low expression, respectively. This latter observation indicates that APRIL has a tumor-promoting role in DLBCL.

\section{Discussion}

We have analyzed APRIL expression in human NHL in situ with specific antibodies allowing identification of APRIL-producing cells, as well as localization of secreted APRIL. We found high numbers of APRIL-producing cells and increased levels of secreted APRIL in about $50 \%$ and $20 \%$ of the DLBCL and BL cases analyzed, respectively. There was no APRIL up-regulation for lower grade B-cell lymphomas. Our analysis at the proteinexpression level is in full accordance with the up-regulation of APRIL gene expression selectively observed in a fraction of DLBCLs by Alizadeh et al. ${ }^{33}$ The cellular source of APRIL in DLBCL lesions was disparate. However, in all the lesions showing APRIL up-regulation, the main source of APRIL was neutrophils. Only one case, likely to be histiocyte-rich DLBCL, ${ }^{34}$ contained a majority of histiocytes. We could not detect tumor cells expressing APRIL. This shows that APRIL expression in tumor cells, reported previously in vitro, ${ }^{16}$ is low compared with in situ neutrophils. The APRIL production by inflammatory cells in B-cell lymphomas likely explains the selective APRIL up-regulation in high-grade B-cell lymphoma lesions, compared with lower grade B-cell lymphoma/leukemia known to develop with a less pronounced host-cell infiltration.

Secreted APRIL was found associated to tumor cells. This localization was ensured by HSPG binding APRIL. The punctuated APRIL/HSPG staining reflects proteoglycan clustering induced on ligand binding. ${ }^{35}$ In addition, APRIL binding to HSPG appears to induce proteoglycan internalization in vivo, because in situ staining for syndecan-4 and secreted APRIL localizes inside the cells. Such internalization of proteoglycan on ligand binding to HSPG has already been described. ${ }^{36}$ Proteoglycan clustering and internalization is likely to indicate an early response to APRIL in DLBCL. HSPG expression by DLBCL tumor cells was confirmed in vitro on cell lines. In addition to proteoglycans, DLBCL tumor cells expressed uniformly BCMA, consistent with previous data. ${ }^{16}$ BCMA staining was low on intact DLBCL tumor cells but significantly enhanced after permeabilization, confirming the presence of a major pool of BCMA in the Golgi apparatus. ${ }^{37}$ In addition to BCMA, some DLBCL tumor cells express TACI, also consistent with previous data. ${ }^{38}$ Hence, APRIL-R expression constitutes another level of heterogeneity in DLBCL tumor cells. HSPG on tumor cells is likely to serve as coreceptor, concentrating the ligand 
on tumor cells. This in situ ligand retention by proteoglycans is efficient, because no circulating APRIL could be detected in the sera of patients with high APRIL expression. Ligand retention may not be the unique role of proteoglycans in APRIL biology. Indeed, secreted APRIL, expected to be a trimer, does not costimulate B cells, unless oligomerized by a crosslinking mAb. ${ }^{21}$ Multiplicity of the binding site on heparan sulfate side chains of proteoglycans is thus likely to mediate such oligomerization of secreted APRIL; this phenomenon has been already reported for some growth factors. ${ }^{39}$ Furthermore, the contribution of HSPG to APRIL signaling may be different, depending on the signaling receptor involved. HSPG, by its capacity to internalize bound ligand, may serve to route APRIL from the extracellular medium toward BCMA expressed in Golgi. For TACI, a direct interaction with HSPG has been recently reported. ${ }^{40}$ In this context, HSPG may form a tight trimolecular complex with cell-surface TACI and APRIL to enhance signaling, as already shown for FGF/FGF-R. ${ }^{41}$ At the present time, it is difficult to state which APRIL signaling receptor is involved. Both are expressed on DLBCL cells depending on the patients. BCMA has been implicated in the survival of post-germinal center plasmablasts. ${ }^{42}$ TACI, despite an early report showing a negative signaling into B cells, ${ }^{43}$ plays also a role in the generation of plasmablasts, ${ }^{44}$ confirmed by a positive signaling transmitted to B cells. ${ }^{40}$ TACI and BCMA are therefore valid candidates, and the specific issue of APRIL receptor(s) involvement in DLBCL warrants further investigations.

Planelles et $\mathrm{al}^{13}$ elegantly showed that transgenic mice overexpressing APRIL in $\mathrm{T}$ cells develop B-cell chronic lymphocytic leukemia (B-CLL)-like lymphoma. This study highlighted the B-cell tumor-promoting role for APRIL in an animal model. We are now showing a correlation between APRIL expression and aggressiveness of human NHL. However, the human situation is different. In human lymphoma lesions, APRIL is mainly produced by inflammatory neutrophils, and strong APRIL expression is restricted to high-grade NHL (DLBCL and BL). The tumorpromoting effect of APRIL observed here supports the currently emerging concept that host inflammatory reactions contribute to tumor development. ${ }^{45}$ APRIL may therefore represent a key molecule in the development of high-grade B-cell lymphoma, in addition to BAFF, also recently associated with NHL aggressiveness. ${ }^{38}$ Prognosis factors commonly used in DLBCL was recently contested by the molecular characterization of DLBCL diversity in gene-expression analyses. ${ }^{2}$ The correlation of APRIL expression and lymphoma aggressiveness reported here indicates that APRIL may be a newly identified biologic factor influencing the development of DLBCL. Investigation with a larger cohort of patients is definitely warranted to assess whether APRIL is able to modulate independently DLBCL development.

\section{Acknowledgments}

We thank E. Roosnek, P. Y. Dietrich, and M. Duchosal for critical reading of the manuscript. The technical expertise of $\mathrm{C}$. Bosshard is greatly acknowledged.

This work was supported by the Dinu Lipatti/Henri DuboisFerrière Foundation and the Louis Jeantet Foundation.

\section{Authorship}

Contribution: J.S., P.M.-F., T. McKee, S.M., T. Matthes, and F.-A.L.G. performed research and analyzed data. P.S., J.T., and O.D. provided valuable validated APRIL-specific reagents. B.H. designed the research, performed research, analyzed data, and wrote the paper.

Conflict-of-interest disclosure: One of the authors (O.D.) is employed by Apotech Corporation, whose product was studied in the present work. The remaining authors declare no competing financial interests.

Correspondence: Bertrand Huard, Louis Jeantet Laboratory, University Medical Center, rue Michel Servet 1, 1211 Geneva 4, Switzerland; e-mail: bertrand.huard@medecine.unige.ch.

\section{References}

1. A clinical evaluation of the International Lymphoma Study Group classification of nonHodgkin's lymphoma. The Non-Hodgkin's Lymphoma Classification Project. Blood. 1997;89: 3909-3918.

2. Gascoyne RD. Emerging prognostic factors in diffuse large B cell lymphoma. Curr Opin Oncol. 2004;16:436-441.

3. Coiffier B, Reyes F, Group d'Etude des Lymphomes de l'Adulte. Best treatment of aggressive non-Hodgkin's lymphoma: a French perspective. Oncology (Williston Park). 2005;19(4 suppl 1):715.

4. Kalled SL, Ambrose C, Hsu YM. The biochemistry and biology of BAFF, APRIL and their receptors. Curr Dir Autoimmun. 2005;8:206-242.

5. Schiemann B, Gommerman JL, Vora K, et al. An essential role for BAFF in the normal development of B cells through a BCMA-independent pathway. Science. 2001;293:2111-2114.

6. Rolink AG, Tschopp J, Schneider P, Melchers F. BAFF is a survival and maturation factor for mouse B cells. Eur J Immunol. 2002;32:20042010.

7. Litinskiy MB, Nardelli B, Hilbert DM, et al. DCs induce CD40-independent immunoglobulin class switching through BLyS and APRIL. Nat Immunol. 2002;3:822-829.

8. He B, Raab-Traub N, Casali P, Cerutti A. EBV- encoded latent membrane protein 1 cooperates with BAFF/BLyS and APRIL to induce T cell-independent Ig heavy chain class switching. J Immunol. 2003;171:5215-5224.

9. Varfolomeev E, Kischkel F, Martin F, et al. APRILdeficient mice have normal immune system development. Mol Cell Biol. 2004;24:997-1006.

10. Castigli E, Scott S, Dedeoglu F, et al. Impaired $\operatorname{IgA}$ class switching in APRIL-deficient mice. Proc Natl Acad Sci U S A. 2004;101:3903-3908.

11. Mackay F, Sierro F, Grey ST, Gordon TP. The BAFF/APRIL system: an important player in systemic rheumatic diseases. Curr Dir Autoimmun. 2005;8:243-265.

12. Mackay F, Tangye SG. The role of the BAFF/ APRIL system in B cell homeostasis and lymphoid cancers. Curr Opin Pharmacol. 2004;4: 347-354.

13. Planelles L, Carvalho-Pinto CE, Hardenberg G, et al. APRIL promotes B-1 cell-associated neoplasm. Cancer Cell. 2004;6:399-408.

14. Batten M, Fletcher $C, N g$ LG, et al. TNF deficiency fails to protect BAFF transgenic mice against autoimmunity and reveals a predisposition to B cell lymphoma. J Immunol. 2004;172: 812-822.

15. Novak AJ, Bram RJ, Kay NE, Jelinek DF. Aberrant expression of B-lymphocyte stimulator by $B$ chronic lymphocytic leukemia cells: a mechanism for survival. Blood. 2002;100:2973-2979.
16. He B, Chadburn A, Jou E, Schattner EJ, Knowles DM, Cerutti A. Lymphoma B cells evade apoptosis through the TNF family members BAFF/BLyS and APRIL. J Immunol. 2004;172:3268-3279.

17. Kern C, Cornuel JF, Billard C, et al. Involvement of BAFF and APRIL in the resistance to apoptosis of B-CLL through an autocrine pathway. Blood. 2004;103:679-688.

18. Klein B, Tarte K, Jourdan M, et al. Survival and proliferation factors of normal and malignant plasma cells. Int J Hematol. 2003;78:106-113.

19. Novak AJ, Darce JR, Arendt BK, et al. Expression of BCMA, TACI, and BAFF-R in multiple myeloma: a mechanism for growth and survival. Blood. 2004;103:689-694.

20. Moreaux J, Legouffe E, Jourdan E, et al. BAFF and APRIL protect myeloma cells from apoptosis induced by interleukin 6 deprivation and dexamethasone. Blood. 2004;103:3148-3157.

21. Ingold K, Zumsteg A, Tardivel A, et al. Identification of proteoglycans as the APRIL-specific binding partners. J Exp Med. 2005;201:1375-1383.

22. Hendriks J, Planelles L, de Jong-Odding J, et al. Heparan sulfate proteoglycan binding promotes APRIL-induced tumor cell proliferation. Cell Death Differ. 2005;12:637-648.

23. Huard B, Arlettaz L, Ambrose C, et al. BAFF production by antigen-presenting cells provides $T$ cell co-stimulation. Int Immunol. 2004;16:467475. 
24. Matthes TW, Kindler V, Leuba F, et al. Optimized entiviral transduction of erythroid precursors from healthy adults and patients with myelodysplastic syndromes. Leukemia. 2002;16:1319-1323.

25. Holler N, Kataoka T, Bodmer JL, et al. Development of improved soluble inhibitors of FasL and CD40L based on oligomerized receptors. J Immunol Methods. 2000;237:159-173.

26. Holler N, Tardivel A, Kovacsovics-Bankowski M, et al. Two adjacent trimeric Fas ligands are required for Fas signaling and formation of a deathinducing signaling complex. Mol Cell Biol. 2003; 23:1428-1440.

27. Mhawech P, Greloz V, Assaly M, Herrmann F. Immunohistochemical expression of 14-3-3 sigma protein in human urological and gynecological tumors using a multi-tumor microarray analysis. Pathol Int. 2005;55:77-82.

28. Lopez-Fraga M, Fernandez R, Albar JP, Hahne M. Biologically active APRIL is secreted following intracellular processing in the Golgi apparatus by furin convertase. EMBO Rep. 2001;2:945-951.

29. Chang CC, Cleveland RP. Decreased CD10-positive mature granulocytes in bone marrow from patients with myelodysplastic syndrome. Arch Pathol Lab Med. 2000;124:1152-1156.

30. Valente AM, Taatjes DJ, Mount SL. Comparison of the pattern of expression of Leu-M1 antigen in adenocarcinomas, neutrophils and Hodgkin's disease by immunoelectron microscopy. Histochem Cell Biol. 1995;103:181-186

31. Flavell DJ, Jones DB, Wright DH. Identification of tissue histiocytes on paraffin sections by a new monoclonal antibody. J Histochem Cytochem. 1987;35:1217-1226.

32. Rosenwald A, Staudt LM. Gene expression profiling of diffuse large B-cell lymphoma. Leuk Lymphoma. 2003;44(suppl 3):S41-S47.

33. Alizadeh AA, Eisen MB, Davis RE, et al. Distinct types of diffuse large B-cell lymphoma identified by gene expression profiling. Nature. 2000;403: 503-511.

34. Wang J, Sun NC, Chen YY, Weiss LM. T-cell/histiocyte-rich large B-cell lymphoma displays a heterogeneity similar to diffuse large B-cell lymphoma: a clinicopathologic, immunohistochemical, and molecular study of 30 cases. Appl Immunohistochem Mol Morphol. 2005;13:109-115

35. Rapraeger A, Jalkanen M, Bernfield M. Cell surface proteoglycan associates with the cytoskeleton at the basolateral cell surface of mouse mammary epithelial cells. J Cell Biol. 1986;103: 2683-2696.

36. Belting M. Heparan sulfate proteoglycan as a plasma membrane carrier. Trends Biochem Sci. 2003;28:145-151.

37. Gras MP, Laabi Y, Linares-Cruz G, et al. BCMAp an integral membrane protein in the Golgi apparatus of human mature B lymphocytes. Int Immunol. 1995;7:1093-1106.

38. Novak AJ, Grote DM, Stenson M, et al. Expression of BLyS and its receptors in B-cell nonHodgkin lymphoma: correlation with disease ac- tivity and patient outcome. Blood. 2004;104: 2247-2253.

39. Wiedlocha A, Sorensen V. Signaling, internalization, and intracellular activity of fibroblast growth factor. Curr Top Microbiol Immunol. 2004;286:4579.

40. Bischof D, Elsawa SF, Mantchev G, et al. Selective activation of TACI by syndecan-2. Blood. 2006;107:3235-3242.

41. Pellegrini L, Burke DF, von Delft F, Mulloy B, Blundell TL. Crystal structure of fibroblast growth factor receptor ectodomain bound to ligand and heparin. Nature. 2000;407:1029-1034.

42. Avery DT, Kalled SL, Ellyard Jl, et al. BAFF selectively enhances the survival of plasmablasts generated from human memory B cells. J Clin Invest. 2003;112:286-297.

43. Seshasayee D, Valdez P, Yan M, Dixit VM, Tumas D, Grewal IS. Loss of TACI causes fatal lymphoproliferation and autoimmunity, establishing TACI as an inhibitory BLyS receptor. Immunity. 2003; 18:279-288

44. Castigli E, Wilson SA, Garibyan L, et al. TACl is mutant in common variable immunodeficiency and IgA deficiency. Nat Genet. 2005;37:829-834.

45. Balkwill F, Coussens LM. Cancer: an inflammatory link. Nature. 2004;431:405-406.

46. Hans CP, Weisenburger DD, Greiner TC, et al. Confirmation of the molecular classification of diffuse large B-cell lymphoma by immunohistochemistry using a tissue microarray. Blood. 2004; 103:275-282. 